\title{
TWO TYPES OF EVOLUTION OF MASSIVE CLOSE
}

\author{
BINAR Y S YSTEMS
}

\author{
C. DE LOORE and J. P. DE GREVE \\ Astrophysical Institute, Vrije Universiteit Brussel, Brussel, Belgium
}

\begin{abstract}
It is well known that the outcome of case B evolution of the primaries of massive close binary systems $\left(M_{1} \geqslant 9 M_{\odot}\right)$ depends on the initial primary mass. The most massive primaries finally ignite carbon, form iron cores and presumably end in a supernova explosion, whereas the lighter ones presumably end as white dwarfs, without carbon ignition. This paper derives an estimate of the mass boundary separating these two kinds of evolution.

As an example of the first case, the evolution of a $20 M_{\odot}+14 M_{\odot}$ system was computed; after the mass exchange, the primary star (with $M=5.43 M_{\odot}$ ) evolves through the helium-burning (Wolf-Rayet) stage towards a supernova explosion; finally the system evolves into an X-ray binary (BWRX-evolution).

As a representative for the second case the evolution of a $10 M_{\odot}+8 M_{\odot}$ system was examined. After the first stage of mass exchange, the primary (with a mass of $1.66 M_{\odot}$ ) approaches the helium main sequence; during later phases of helium burning the radius increases again, and a second stage of mass transfer starts; after this the star (with a mass of $1.14 M_{\odot}$ ) again evolves towards the left in the Hertzsprung-Russell diagram and ends as a white dwarf (BSWD-evolution). A system of $15 M_{\odot}+8 M_{\odot}$ is found to evolve very similar to the $20 M_{\odot}+14 M_{\odot}$ system. The mass $M_{u}$, separating the two types of evolution, must therefore be situated between 10 and 15 solar masses. An initial chemical composition $X=0.70, Z=0.03$ was used for all systems.
\end{abstract}

\section{Introduction}

During the last years supporting evidence has been given (Mikulásek, 1969; Smith, 1973) to Paczynski's hypothesis $(1967,1968)$ that Wolf-Rayet binaries are the evolutionary products of massive close binary systems, attained through a case B of mass exchange. Computations of Tutukov et al. (1974), De Loore and De Grève (1975), De Loore et al. (1975) strongly suggest that this stage is an intermediate step towards the massive X-ray binaries, a fact that was suggested by Van den Heuvel and Heise (1972) and Van den Heuvel (1973). According to these computations primary ancestors of these sources must have been larger than about $15 M_{\odot}$.

On the other hand binary systems with primaries in the range $M<3 M_{\odot}$ are known to evolve into systems with a helium white dwarf component less massive than $0.4 M_{\odot}$ (Kippenhahn et al., 1967; Refsdal and Weigert, 1970; Paczynski, 1970a). Systems with initial primary masses between about $3 M_{\odot}$ and about $8 M_{\odot}$ leave helium stars with masses below about $1.44 M_{\odot}$, which also are likely to finish as white dwarfs. This holds for an initial composition $X=0.70, Z=0.03$; for lower $X$-values the boundaries of this mass range shift to slightly higher masses. So the boundary for systems that leave a white dwarf or evolve towards a supernova explosion is roughly expected to be situated somewhere between about $8 M_{\odot}$ and $15 M_{\odot}$.

The present paper is concerned with a more accurate determination of the bifurcation point between these two types of evolution, designated here as BWRX and BSWD.

In view of the uncertainties connected with carbon ignition in the center and the subsequent evolution (Arnett, 1974; Paczynski, 1974) attention was paid to the evolution of the core. We assumed that stars with helium cores between 4-8 $M_{\odot}$ ignite carbon non explosively (Paczynski, 1971a) and end as a neutron star due to the implosion of the 
iron core. Stars with cores smaller than $1.4 M_{\odot}$ end as white dwarfs (Kippenhahn et al., 1967; Paczynski, 1971b), while the fate of stars with cores between $1.4 M_{\odot}$ and $4 M_{\odot}$ is not so surely determined.

We considered two groups of systems. For the first group (case B - Wolf-Rayet X-ray source, called BWRX) the evolution of a system with a primary of $20 M_{\odot}$ was used as a typical example for the mass range above $20 M_{\odot}$. For the second group (case B - second stage - white dwarf, called BSWD) the evolution of a system with a $10 M_{\odot}$ primary was computed through two separated stages of mass exchange up to the white dwarf stage (De Grève and De Loore, 1976). These computations complete and exceed the sequence computed by Ziołkowski (1974). Unfortunately computations for systems with masses between 10 and $20 M_{\odot}$ do not extend far enough to decide to which group the systems belong (Paczynski, 1967). Therefore calculations were also performed for a system with a primary of $15 M_{\odot}$. The chemical composition of the initial systems was a Population I composition: $X=0.7, Z=0.03$ (Stothers, 1972, 1973). In all three sequences the case $B$ mass transfer was assumed to be conservative (total mass and angular momentum remain constant).

In Section 2 the general features of the evolution of the considered systems are briefly outlined. In Section 3 some parameters which determine the bifurcation in evolution are discussed and some conclusions are given. The most relevant features of the evolution of the three systems are given in Table I, and the evolutionary paths are shown in Figure 1.

\section{The Computed Evolutionary Sequences}

\subsection{SYSTEM WITH A $20 M_{\odot}$ PRIMARY}

The evolution of systems consisting of a primary of $20 M_{\odot}$ and secondaries of respectively 14, 10, 8 and $6 M_{\odot}$ was computed already and published previously (De Loore and De Grève, 1975). The evolution is mainly as outlined by Van den Heuval and Heise (1972). The primary passes through a helium burning helium star stage (presumably Wolf-Rayet) after the mass transfer, ignites carbon non-explosively (Paczynski, 1971a) and finally explodes as a supernova (Arnett, 1973). Systems like these and others computed by Paczynski (1967), Kippenhahn (1969), Barbaro et al. (1969) and Tutukov et al. (1974) are thought to be the ancestors of massive X-ray binaries (Van den Heuvel, 1973; De Loore et al., 1975).

\subsection{SYSTEM WITH A $10 M_{\odot}$ PRIMARY}

The computations were performed for a $10 M_{\odot}+8 M_{\odot}$ system. The first part of the evolution proceeds in a manner similar to that of Ziołkowski's results (1974) for his model of $\beta$ Lyr. However, further calculations show that $3.16 \times 10^{6} \mathrm{yr}$ after the end of the first mass transfer stage, the primary of $1.66 M_{\odot}$ again expands beyond its Roche lobe and a second stage of mass transfer starts (De Grève and De Loore, 1976). The mass drops below $1.2 M_{\odot}$ and ignition of carbon does not occur. Due to the lack of nuclear energy production in the degenerate core and the extinction of the thin helium shell source the star shrinks rapidly and finally settles as a white dwarf.

\subsection{SYSTEM WITH A $15 M_{\odot}$ PRIMARY}

A $15 M_{\odot}+8 M_{\odot}$ system was investigated up to carbon ignition in the core. In order to 
TABLE I

Main characteristics in the evolution of 3 massive close binary systems evolving through a case $B$ of mass transfer

\begin{tabular}{|c|c|c|c|c|c|c|c|c|c|c|c|c|}
\hline & \multicolumn{4}{|l|}{$10+8$} & \multicolumn{4}{|c|}{$15+8$} & \multicolumn{4}{|c|}{$20+14$} \\
\hline & $P$ & $M_{1}$ & $M_{2}$ & $\frac{t}{10^{6} \mathrm{yr}}$ & $P$ & $M_{1}$ & $M_{2}$ & $\frac{t}{10^{6} \mathrm{yr}}$ & $P$ & $M_{1}$ & $M_{2}$ & $\frac{t}{10^{6} \mathrm{yr}}$ \\
\hline Initial system & 3.15 & 10 & 8 & 0 & 4.96 & 515 & 8 & 0 & 4.87 & 720 & 14 & 0 \\
\hline $\begin{array}{l}\text { Start first stage } \\
\text { of mass transfer }\end{array}$ & 3.15 & 10 & 8 & 15.149 & 4.96 & 515 & 8 & 8.403 & 4.87 & 20 & 14 & 6.155 \\
\hline $\begin{array}{l}\text { End first stage } \\
\text { of mass transfer }\end{array}$ & 81.07 & 1.66 & 16.34 & 15.310 & 31.09 & 3.30 & 19.70 & 8.438 & 28.58 & 5.43 & 328.57 & 6.174 \\
\hline $\begin{array}{l}\text { Carbon ignition } \\
\text { Start second } \\
\text { stage of mass } \\
\text { transfer }\end{array}$ & 81.07 & 1.66 & 16.34 & 18.474 & 31.09 & 93.30 & 19.70 & 9.434 & 28.58 & 85.43 & 328.57 & 6.746 \\
\hline $\begin{array}{l}\text { End second } \\
\text { stage of } \\
\text { mass transfer } \\
\text { Supernova }\end{array}$ & 226.16 & 1.14 & 16.86 & 18.512 & & & & & & & & \\
\hline $\begin{array}{l}\text { Supernova } \\
\text { Configuration }\end{array}$ & & hite dy & warf & & & & & & 33.53 & 32 & 28.57 & 6.766 \\
\hline $\begin{array}{l}\text { Configuration } \\
\text { at the end of } \\
\text { the computa- } \\
\text { tions }\end{array}$ & & quenc & main & & & $\begin{array}{l}\text { massiv } \\
\text { sequen }\end{array}$ & e main & & & $\begin{array}{l}\text { Binary } \\
\text { two rur } \\
\text { neutror }\end{array}$ & $\begin{array}{l}\text { pulsar } \\
\text { on away } \\
\text { n stars }\end{array}$ & \\
\hline
\end{tabular}

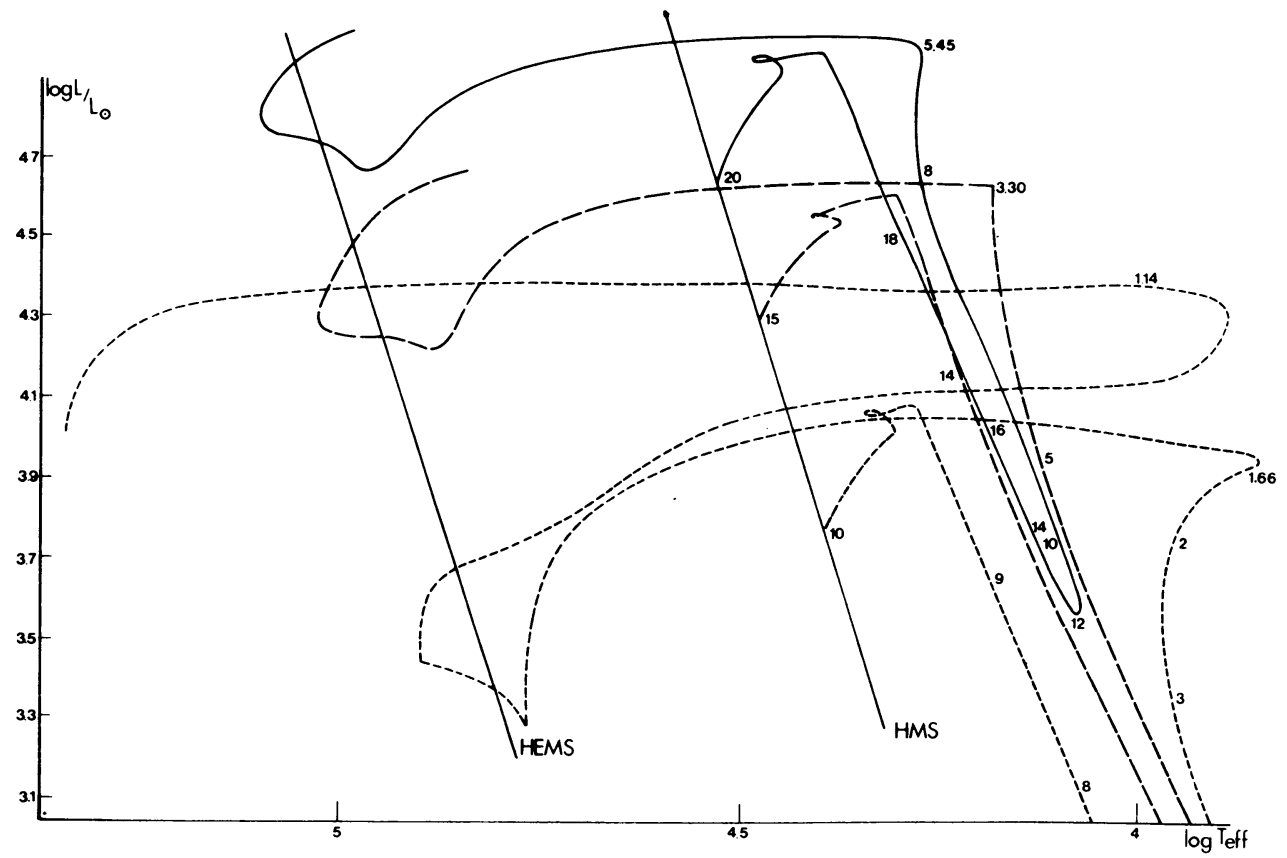

Fig. 1. Evolution tracks for the primaries of three binary systems: (a) $20 M_{\odot}+14 M_{\odot}$ (full line); (b) $15 M_{\odot}+8 M_{\odot}$ (dashed line); and (c) $10 M_{\odot}+8 M_{\odot}$ (dotted line).

The hydrogen (HMS) and helium (HEMS) main sequences are indicated. The numbers refer to the masses. For simplicity only the curves with changing atmospheric composition are given (see text). 
verify that no second stage of mass transfer can occur for this system the influence of the chemical composition of the envelope on the expansion of the star was examined. Computations for two possible configurations of the atmosphere were performed:

(i) the outer layers adopt the composition of the outermost layer of the interior model (helium core model);

(ii) once the first mass transfer is finished the primary star retains its hydrogen rich atmosphere $(X \sim 0.2)$.

In both cases the primary ignites carbon well before the radius equals the Roche radius. Once this was found the further evolution of the system was assumed to occur according to the BWRX scheme.

We emphasize here that the luminosities of the systems (b) and (c) during core helium burning ( $\log L \approx 3.4$ for (b), and $\log L \approx 4.2$ for (c)) remain well below the values of the observed WR stars in binary systems $(\log L>4.6$; Smith 1973).

\section{Behaviour of the Interior}

The behaviour of the central temperature and density of the three computed models is shown in Figure 2, together with available data of the evolution of single stars. The dashdotted line is the boundary between the non-degenerate and the degenerate region. The curve $\left(\epsilon_{\nu}=\epsilon_{\mathrm{cc}}\right)$ marks the onset of carbon burning, according to Paczynski (1967). He found that normal evolving stars with masses between 10 and $15 M_{\odot}$ develop carbon cores with masses ranging from $2.3 M_{\odot}$ to $3.9 M_{\odot}$. In this case the temperature increases with increasing density until carbon ignition is attained.

Furthermore it has been shown that the behaviour of case B mass exchange is rather independent of the core mass (Plavec, 1968; Ziołkowski, 1970) and is finally stopped by core helium ignition. The mass of the remnant is roughly the mass of the helium core and further evolution may be compared with helium stars of the same mass computed by Paczynski (1971a) and Arnett $(1973,1974)$. This picture can only be altered if the mass of the remnant is changed again during a second mass transfer stage. According to our computations this does not occur for primary masses equal to or larger than $15 M_{\odot}$.

Figure 3 shows the evolution of the inner layers of the remnant of the initial $10 M_{\odot}$ primary during core helium burning and the second stage of mass loss, i.e. extent in mass and radius of the convective core, the hydrogen exhausted layers and the carbon core as a function of time. Immediately after the second stage, terminated by the end of the shell burning, the star shrinks rapidly. Subsequent evolution may be derived by interpolation between the tracks of Paczynski (1970b) for cores of $0.8 M_{\odot}$ and $1.2 M_{\odot}$ and the track of Rose and Smith (1970) for a $0.85 M_{\odot}$ star.

In Figure 4 the behaviour of the core of the remnant of the initial $20 M_{\odot}$ primary is shown during core helium burning, up to carbon ignition. The hydrogen envelope in the $5.43 M_{\odot}$ remnant of $20 M_{\odot}$ is relatively thin; it contains $42 \%$ of the total mass compared with $60 \%$ in the $1.66 M_{\odot}$ remnant of $10 M_{\odot}$. After helium exhaustion in the core the outer layers expand at a much lower rate in the $5.43 M_{\odot}$ star than in the $1.66 M_{\odot}$ star; the chemical composition of the envelopes is the same for both remnants during this stage. Note also that in the $5.43 M_{\odot}$ star the mass of the carbon core always remains smaller than the largest value of the convective core, while this is not the case in the $1.66 M_{\odot}$ star. 


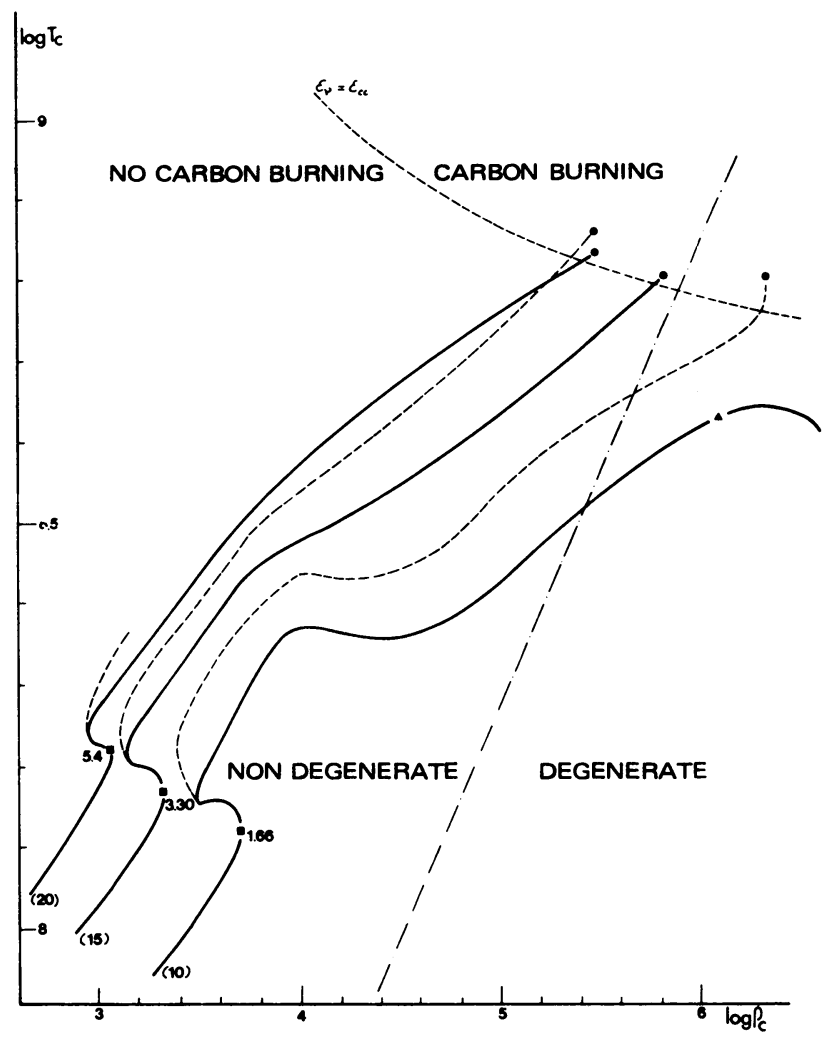

Fig. 2. $\log \rho_{\mathrm{c}}$ versus $T_{\mathrm{c}}$ diagram for the three computed systems, from approximately the end of the mass exchange till the end of the computations (full lines). For comparison similar tracks for single stars (De Loore, 1972) are given (dashed lines). The numbers indicate the total mass of the star. The squares mark the end of the mass transfer $\left(20 M_{\odot}\right.$ and $\left.15 M_{\odot}\right)$ or the end of the first mass transfer stage $\left(10 M_{\odot}\right)$. For the latter case the end of the second mass transfer phase is indicated by a triangle. The end of the computations is marked by dots.

From the analysis of the evolution of the three systems we can draw the following conclusions:

(1) Systems with primaries in the range roughly from $9 M_{\odot}$ (a discussion of the lower masses is beyond the scope of this paper) to $M_{u}$ are expected to pass through a second stage of mass transfer and are likely to end as systems with a white dwarf member. The value of $M_{u}$ is situated between $10 M_{\odot}$ and $15 M_{\odot}$, for an initial composition $X=0.7$, $Z=0.03$.

(2) Systems with primaries more massive than $M_{u}$ pass through a supernova explosion and form neutron stars or black holes.

(3) The observed high luminosities confirm, in view of our evolutionary computations, that systems with original primaries smaller than $15 M_{\odot}$ cannot produce WR binaries (Paczynski, 1971b; De Grève and De Loore, 1975).

(4) As a consequence of the faster evolution of massive stars and of the second stage of mass transfer, white dwarfs originating from massive primaries are formed in a much 


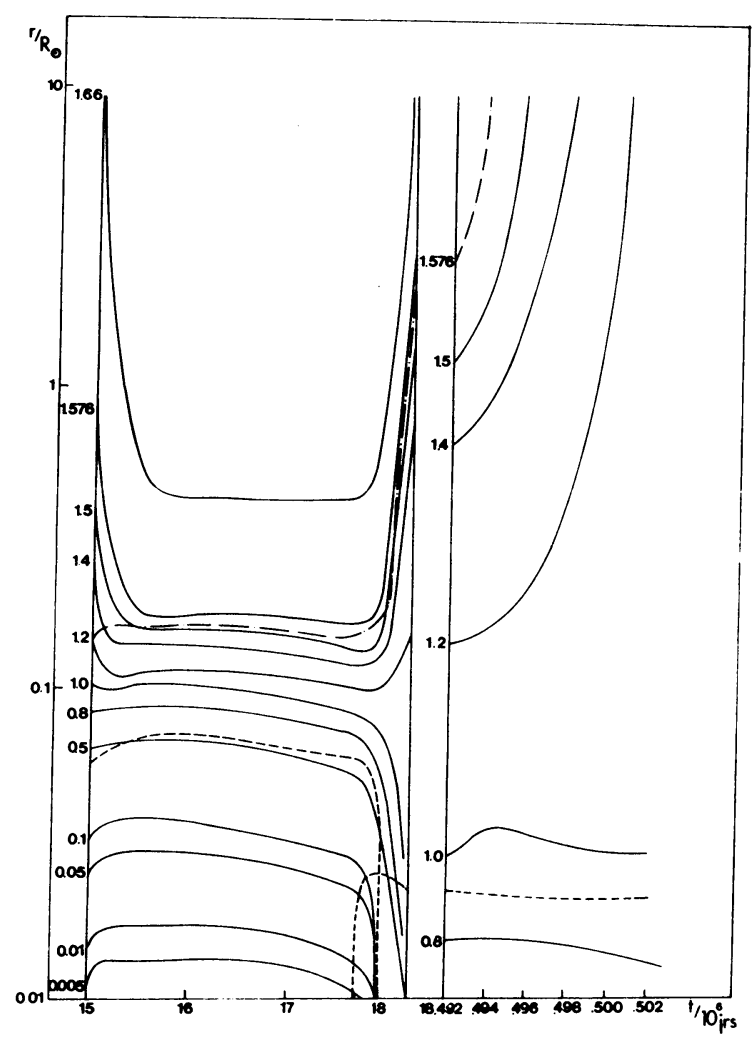

Fig. 3. The changes in the inner structure of the primary in the system $10 M_{\odot}+8 M_{\odot}$ in the course of helium shell burning and during the second stage of mass transfer. The corresponding absolute mass fraction (full line) is indicated for each shell. The boundary of the convective core is given by a dashed line. Dash dotted lines limit the hydrogen exhausted layers, the dotted line the carbon core.

shorter time than those arising from lower mass stars. For our $10 M_{\odot}+8 M_{\odot}$ system the total evolution time from main sequence primary to white dwarf is $1.851 \times 10^{7} \mathrm{yr}$, while for a $2 M_{\odot}+1.5 M_{\odot}$ system (Giannone and Gianuzzi, 1970) the evolution for various initial periods varies between $6.659 \times 10^{8}$ and $7.049 \times 10^{8} \mathrm{yr}$.

\section{References}

Arnett, W. D.: 1973, in D. Schramm and W. D. Arnett (eds.), Explosive Nucleo-Synthesis, University of Texas Press, London, England.

Arnett, W. D.: 1974, in R. J. Tayler (ed.), 'Late Stages of Stellar Evolution', IAU Symp. 66, 1.

Barbaro, G., Giannone, P., Gianuzzi, M. A., and Summa, C.: 1969, in M. Hack (ed.), Mass Loss from

Stars, Second Trieste Colloquium on Astrophysics, D. Reidel, Dordrecht, Holland, p. 217.

De Grève, J. P. and De Loore, C.: 1975, Astron. Astrophys. 42, 91.

De Grève, J. P. and De Loore, C.: 1976, Astrophys. Space Sci. (in press).

De Loore, C.: 1972 (unpublished).

De Loore, C. and De Grève, J. P.: 1975, Astrophys. Space Sci. 35, 241.

De Loore, C., De Grève, J. P., and De Cuyper, J. P.: 1975, Astrophy's. Space Sci. 36, 219.

Giannone, P. and Gianuzzi, M. A.: 1970, Astron. Astrophys. 6. 309. 


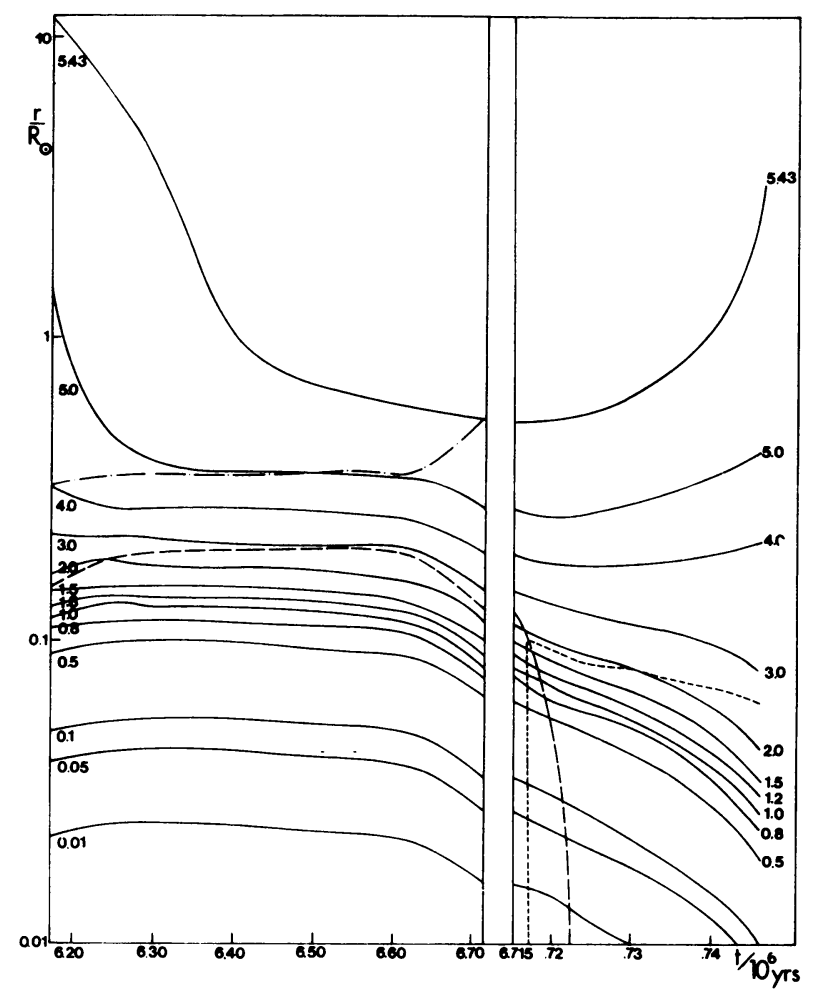

Fig. 4. The change in the inner structure of the primary in the system $20 M_{\odot}+14 M_{\odot}$ up to carbon 1gnition. The notation is the same as in Figure 3.

Kippenhahn, R.: 1969, Astron. Astrophys. 5, 571.

Kippenhahn, R., Kohl, K., and Weigert, A.: 1967, Z. Astrophys. 66, 58.

Mikulásek, Z.: 1969, Bull. Astron. Inst. Czech. 20, 4.

Paczynski, B.: 1967, Acta Astron. 17, 355.

Paczynski, B.: 1968, in L. Perek (ed.), Highlights in Astronomy, Proc. 13th General Assembly, IAU,

D. Reidel, Dordrecht, Holland, p. 409.

Paczynski, B.: 1970a, Acta Astron. 20, 47.

Paczynski, B.: 1970b, Acta Astron. 20, 195.

Paczynski, B.: 1971a, Acta Astron. 21, 1.

Paczynski, B.: 1971b, Ann. Rev. Astron. Astrophys. 9, 183.

Paczynski, B.: 1974, in R. J. Tayler (ed.), 'Late Stages of Stellar Evolution', IAU Symp. 66, 62.

Plavec, M.: 1968, Adv. Astron. Astrophys. 6, 201.

Refsdal, S. and Weigert, A.: 1970, Astron. Astrophys. 6, 426.

Rose, W. K. and Smith, R. L.: 1970, Astrophys. J. 159, 903.

Smith, L. F.: 1973, in M. K. V. Bappu and J. Sahade (eds.), 'Wolf-Rayet and High-Temperature Stars', IAU Symp. 49, 15.

Stothers, R.: 1972, Astrophys. J. $175,715$.

Stothers, R.: 1973, Astrophys. J. 184, 181.

Tutukov, A. V., Yungel'son, L. R., and Kraitcheva, Z. T.: 1974, Proceedings of the 2nd IAU Regional Meeting, Trieste.

Van den Heuvel, E. P. J.: 1973, Nature, Phys. Sci. 242, 71.

Van den Heuvel, E. P. J., and Heise, J.: 1972, Nature, Phys. Sci. 239, 67.

Ziołkowski, J.: 1970, Acta Astron. 20, 213.

Ziołkowski, J.:1974, Bull. Amer. Astron. Soc. 6, 263. 


\section{DISCUSSION}

Eggleton: In the case of the first mass transfer phase, is there not a possibility that the secondary will swell up by accretion to such an extent that the two stars come into contact? This gives a big uncertainty to later stages.

De Loore: A precise treatment of the evolution of the mass accreting star has not yet been performed; we computed it in a very simple way, just by supposing that matter rains down on the secondary without interaction. A more sophisticated computation has been done by Benson in case A. Probably for the case B situation we examine here there is no risk that the mass accreting star swells and will loose mass in the reverse sense. Our computations for mass accreting stars show that such a star follows the hydrogen main sequence (as is normal since the star is gaining hydrogen) and afterwards evolves into a blue supergiant stage, which is perfectly compatible with X-ray observations.

Paczynski: The only available results indicate that contact cannot be avoided in case $A$ of mass exchange. Nothing is known about case B. I hope contact can be avoided as the system is large in case B.

Hilditch: Is there any chance for further interaction from the secondary before the primary reaches the white dwarf stage?

De Grève: After the mass exchange the secondary was followed as if it were a normal $16 M_{\odot}$ main sequence star until it overflowed its Roche lobe. This occurred long after the primary reached the white dwarf stage, due to the large time the star spends on the main sequence.

Whelan: Are there initial conditions in which the second mass exchange starts before the old primary has become a white dwarf or neutron star? If there are the supernova may not occur.

De Loore: I think it is possible if the masses of the components do not differ too much. We examined the case of two components of nearly equal masses, and tried to compute their evolution with mass transfer to and fro but we did not succeed, i.e. we ended in a contact system. So we cannot say if these conditions are realistic or not, but it is possible that such systems might exist. If mass loss from the systems is taken into account the situation changes. 